$(\mathrm{aOR}=2.35 ;$ IC 95\%: 1.02-5.44), whose monthly income decreased $(\mathrm{aOR}=13.61$; IC 95\%: 1.49-124.35) or persisted in the lowest income range $(\mathrm{aOR}=14.56$; IC 95\%: 1.46-144.89). This was also the case for those workers who continued their temporary documented $(\mathrm{aOR}=5.05$; IC 95\%: 1.39-18.34) or undocumented $(\mathrm{aOR}=36.59$; IC 95\%: 2.47-541.17) status. A decreased risk was observed in workers who became affiliated to the Social Security (aORa $=0.10$; IC 95\%: 0.02-0.48).

Conclusions There is an increase of poor mental health in immigrant workers in Spain related to the worsening of their employment conditions. At the same time, it was observed a decrease when Social Security protection increased.

\section{AFTER HURRICANE SANDY, IMPLEMENTATION OF THE EXPANDED SYNDROMIC SURVEILLANCE FOR RECOVERY WORKERS AND RESIDENTS}

${ }^{1}$ H K Kim, 'Liu, ${ }^{2}$ Tak, 'Dropkin, 'Moline, ' ${ }^{1}$ Silverman. 'Hofstra North Shore-LIJ School of Medicine, Great Neck, NY, United States of America; ${ }^{2}$ Massachusetts Department of Public Health, Boston, United States of America

\subsection{6/oemed-2013-101717.209}

Objectives Hurricane Sandy brought wide spread devastation in the region of New York and New Jersey in USA. To our knowledge, there have been no official reports of the health effects from Hurricane Sandy since the day of Hurricane arrival 10/29 in 2012.

Methods To rapidly assess the health impacts of Hurricane Sandy among recovery workers and residents in the affected area, we are building an expanded syndromic surveillance system that integrates the near real-time electronic Emergency Department (ED) visit records collected from 14 EDs within a large health system in NY, the air quality index data from the Environmental Protection Agency (EPA), and the weather data from the National Oceanic and Atmospheric Administration (NOAA). Recovery workers will be identified from billing database. Approximately $50 \%$ of occupational/industrial information of ED patients are missing, however patients with work-related injuries and illnesses required to provide this information according to Worker's Compensation system in US. Through a multilevel case-crossover design, we aim to rapid identification of elevated health effects during four exposure windows we developed (the Pre-, During-, Short-term, and Long-term PostHurricane) by comparing ED records before (from 2005) and after Hurricane Sandy while adjusting for air pollution levels and weather conditions. The continuing monitoring during the fourth exposure window (i.e. Long-term Post- Hurricane) will ensure early detection of potential occurrences of chronic diseases stemmed from the initial disaster-related acute forms. The implementation of the syndromic surveillance within a large health system will not only improve the healthcare delivery, but also provide important information to the outside stakeholders such as public health agencies to enhance strategic planning for rapid post-disaster response.

\section{ATTENUATION OF EXPOSURE EFFECTS OVER TIME: A SIMULATION STUDY}

${ }^{1} \mathrm{~K}$ Steenland, ${ }^{2}$ Karnes, ${ }^{2}$ Barry, ${ }^{2}$ Darrow. ${ }^{1}$ Atlanta, United States of America; ${ }^{2}$ Emory U, Atlanta, Ga, United States of America

10.1136/oemed-2013-101717.210
Background Positive exposure-response trends often diminish at higher cumulative exposure, correlated with longer follow-up time. Depletion of susceptibles, increased measurement error at higher cumulative exposure, and saturation of biological pathways, have all been postulated as reasons for attenuation.

Methods We conducted simulations to evaluate rate ratios over time under different assumptions about susceptibility to exposure effects and measurement error; we evaluated exposure-response trends to determine whether attenuation was evident. We simulated a dynamic cohort in which entry occurred over time; the metric of interest was duration of exposure. We also considered cross-sectional analyses in which follow-up started only after a certain point of time. Simulations considered 10,000 subjects enrolled from 1940-2010 and followed through 2020. Ten simulations were conducted for each scenario and exposureresponse parameters averaged. An excess relative risk model was used to generate the relationship between duration of exposure and disease, controlling for age. Measurement error of both classical and Berkson type were simulated, with increasing error with increasing exposure. Cox regression was used to evaluate exposure-response trends.

Results Under all scenarios considered with less than 100\% susceptibility among the exposed, there was evidence of depletion of susceptibles over follow-up time. However, under realistic scenarios considered here, there was only modest evidence of attenuation of a linear exposure-response trend due to depletion of susceptibles. Classical measurement error, but not Berkson error, produced attenuation. Cross-sectional analyses did not dramatically change attenuation patterns.

Conclusions Marked attenuation of exposure-response trends over follow-up time is more likely due saturation of biological pathways or, perhaps less likely, to classical measurement error than to either the depletion of susceptibles or Berkson measurement error.

\section{A NEW OCCUPATIONAL CANCER: CHOLANGIOCARCINOMA AMONG OFFSET COLOR PROOF-PRINTING WORKERS EXPOSED TO 1,2- DICHLOROPROPANE AND/OR DICHLOROMETHANE}

${ }^{1} \mathrm{~S}$ K Kumagai, ${ }^{2}$ Kurumatani. 'University of Occupational and Environmental Health, Kitakyushu, Japan; ${ }^{2}$ Nara Medical University School of Medicine, Kashihara, Japan

\subsection{6/oemed-2013-101717.211}

Objectives At the 85th Annual Meeting of the Japan Society for Occupational Health (31 May, 2012, Nagoya, Japan), we reported a cluster of five patients with cholangiocarcinoma among workers in the offset colour proof-printing section of a small printing company in Osaka, Japan. This was the first report of occupational cholangiocarcinoma in the world. Further investigation led to the identification of a total of 11 patients (Occup Environ Med 2013, in print). Here, we present an epidemiological characteristic of the workers in the printing section.

Methods We identified 62 men who had worked in the proofprinting section for at least one year between 1991 and 2006. We interviewed them to learn about the chemicals they used, and estimated their chemical exposure levels. We also investigated medical records of the 11 patients and calculated the standardised mortality ratio (SMR) from 1991 to 2011.

Results Workers used 1,2-dichloropropane (1,2-DCP) from approximately 1985 to 2006 and dichloromethane (DCM) from approximately 1985 to $1997 / 98$. Exposure concentrations were estimated to be 100-670 ppm for 1,2-DCP and 80-540 ppm for 